\title{
Creation of Dirac monopoles in spinor Bose-Einstein condensates
}

\author{
Ville Pietilä ${ }^{1,2}$ and Mikko Möttönen ${ }^{1,2,3}$ \\ ${ }^{1}$ Department of Applied Physics/COMP, Helsinki University of Technology P. O. Box 5100, FI-02015 TKK, Finland \\ ${ }^{2}$ Australian Research Council, Centre of Excellence for Quantum Computer Technology, \\ The University of New South Wales, Sydney 2052, Australia \\ ${ }^{3}$ Low Temperature Laboratory, Helsinki University of Technology, P. O. Box 3500, FI-02015 TKK, Finland
}

\begin{abstract}
We demonstrate theoretically that using standard external magnetic fields, one can imprint point-like topological defects to the spin texture of a dilute Bose-Einstein condensate. Symmetries of the condensate order parameter render this topological defect to be accompanied with a vortex filament corresponding to the Dirac string of a magnetic monopole. The vorticity in the condensate coincides with the magnetic field of a magnetic monopole, providing an ideal analogue to the monopole studied by Dirac.
\end{abstract}

PACS numbers: 03.75.Lm,03.75.Mn

The existence of particles with non-zero magnetic charge, that is, magnetic monopoles has far-reaching implications to the laws of quantum mechanics, theories of elementary particles, and cosmology [1, 2, 3]. However, experimental evidence of magnetic monopoles as fundamental constituents of matter is still absent, and hence there is great incentive to search corresponding configurations in experimentally more tractable systems. Several aspects of monopoles have been investigated in the context of liquid crystals [4], anomalous quantum-Hall effect [5], and exotic spin systems [6]. Recently, Qi et al. proposed that a magnetic monopole could be induced in a topological insulator [7]. Despite these elegant proposals, an experimental realization of a magnetic monopole as an emergent particle or any analog of the Dirac monopole in still lacking. One of the most promising candidate systems to realize such analogies has been superfluid ${ }^{3} \mathrm{He}[8$, 9, 10, 11], but to date there are no direct experimental observations of these topological excitations.

Dilute Bose-Einstein condensates (BECs) of alkali atoms with a hyperfine spin degree of freedom combine magnetic and superfluid order and share many common features with the superfluid ${ }^{3} \mathrm{He}[11,12,13,14]$. The order parameter describing such systems is typically invariant under global symmetries that form a non-Abelian group and monopoles and other textures can occur if this symmetry is broken. On the other hand, spinor BECs are well-suited to host artificially generated gauge fields which can provide an alternative method to realize a magnetic monopole [15]. In the simplest case of spin- 1 condensate, a variety of different topological defects such as global monopoles [16], non-Abelian magnetic monopoles [17], global textures [18] and in particular, analogies to the Dirac monopole [19] have been investigated. In the related two-component condensates, skyrmion textures have been studied by several authors [20, 21, 22]. An experimental realization of any of these topological states still remains a milestone in the field of cold atoms.

In this Letter, we consider a spinor BEC with the total hyperfine spin $F=1$ which in the absence of external magnetic fields has two phases: a ferromagnetic and an antiferromagnetic (polar) phase [13]. However, in the presence of a strong enough external magnetic field, the spin of the order parame- ter aligns with the local field and the condensate order parameter corresponds to the ferromagnetic phase. Modifying the external field adiabatically, multi-quantum vortices can be imprinted into the condensate as was proposed theoretically by Nakahara et al. [23] and verified experimentally by Leanhardt et al. [24]. The method we employ here utilizes the same ideology in this respect, but due to non-trivial three-dimensional structure of the magnetic field we are able to create a pointlike defect to the spin texture of the condensate that gives rise to vorticity coinciding with the magnetic field of a magnetic monopole.

Let us assume first that the hyperfine spin of the condensate is aligned with an external magnetic field which is a combination of two quadrupole fields and a homogeneous bias field

$$
\boldsymbol{B}(\boldsymbol{r}, t)=B_{1}^{\prime}(x \hat{\boldsymbol{x}}+y \hat{\boldsymbol{y}})+B_{2}^{\prime} z \hat{\boldsymbol{z}}+B_{0}(t) \hat{\boldsymbol{b}},
$$

where Maxwell's equations impose the condition $2 B_{1}^{\prime}+B_{2}^{\prime}=$ 0 for the magnetic field gradients. The direction of the bias field is determined by the unit vector $\hat{b}$. Such combination of quadrupole fields is produced by, e.g., a crossing pair of Helmholtz coils, and it has recently been proposed to dynamically generate knot-like textures in antiferromagnetic BECs [18]. The point where the external magnetic field vanishes is the center of a monopole defect in the spin texture $\mathcal{S}=\boldsymbol{\Psi}^{\dagger} \mathcal{F} \boldsymbol{\Psi}$ given by the condensate order parameter $\boldsymbol{\Psi}=$ $\left(\psi_{1}, \psi_{0}, \psi_{-1}\right)^{T}$ and the spin-1 matrices $\mathcal{F}=\left(\mathcal{F}_{x}, \mathcal{F}_{y}, \mathcal{F}_{z}\right)$. This monopole can be characterized by the charge [19]

$$
\mathcal{Q}=\frac{1}{8 \pi} \int_{\Sigma} \mathrm{d}^{2} \sigma_{i} \varepsilon_{i j k} \varepsilon_{a b c} \hat{s}_{a} \partial_{j} \hat{s}_{b} \partial_{k} \hat{s}_{c}
$$

where $\hat{s}=\mathcal{S} /|\mathcal{S}|$ and the integral is taken over a surface $\Sigma$ enclosing the defect. The indices take values $1,2,3$ and summation over repeated indices is implied. The Levi-Civita tensor is denoted by $\varepsilon_{\alpha \beta \gamma}$. For the magnetic field in Eq. (1), the charge of the spin texture becomes $\mathcal{Q}=1$. A schematic illustration of two possible textures is shown in Fig. 1. For the ferromagnetic order parameter manifold, the second homotopy group is trivial and isolated monopole defects are not allowed. Thus the monopole defect in the spin texture is associated with a vortex filament extending outwards from the 
(a)

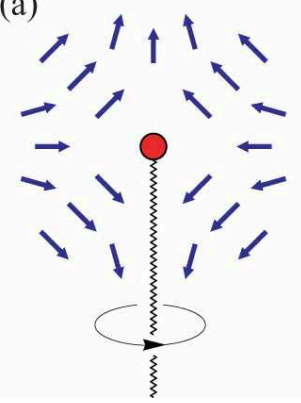

(b)

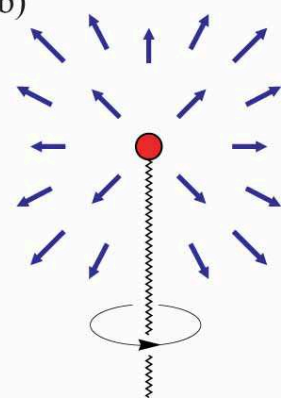

FIG. 1: Possible configurations for the spin texture. Both configurations are characterized by the same charge $\mathcal{Q}=1$ but only the one in (a) can be imprinted using the combination of two quadrupole fields. The hedgehog texture in (b) describes the vorticity $\boldsymbol{\Omega}_{s}$ corresponding to the spin texture in panel (a). In both cases, the vector fields are symmetric with respect to rotations about the axis on which the singularity filament (zigzag line) lies.

monopole giving rise to a physical Dirac string [1, 19]. Since the monopole is located at the zero of the magnetic field, adjusting the bias field $B_{0}(t)$ moves the monopole along the axis given by $\hat{b}$. In particular, the monopole can be brought in from outside of the condensate by taking large enough bias to begin with and then ramping it down adiabatically.

The superfluid velocity can be defined as $\boldsymbol{v}_{s}=$ $-i \hbar \zeta^{\dagger} \nabla \zeta / m$, where $\boldsymbol{\Psi}=\sqrt{\varrho} \zeta, \varrho$ is the density of particles, and $m$ is the mass of the constituent atoms [13, 19, 25]. For simplicity, we first assume that $\hat{\boldsymbol{b}}=\hat{\boldsymbol{z}}$ and define new coordinates by $\left(x^{\prime}, y^{\prime}, z^{\prime}\right)=\left(x, y, 2 z-B_{0}(t) / B_{1}^{\prime}\right)$. In the adiabatic limit, the condensate order parameter corresponds to the local eigenstate of the linear Zeeman operator $g_{F} \mu_{B}|\boldsymbol{B}| \hat{\boldsymbol{n}} \cdot \mathcal{F}$ with $\hat{\boldsymbol{n}}=(\sin \beta \cos \alpha, \sin \beta \sin \alpha, \cos \beta)$. The local Zeeman state can be written as

$$
|\zeta(\alpha, \beta)\rangle=\mathcal{U}(\alpha, \beta)\left|F, m_{F}\right\rangle,
$$

where the unitary transformation $\mathcal{U}(\alpha, \beta)$ is given by $\mathcal{U}(\alpha, \beta)=e^{-i \alpha \mathcal{F}_{z}} e^{-i \beta \mathcal{F}_{y}} e^{i \alpha \mathcal{F}_{z}}$ and the state $\left|F, m_{F}\right\rangle$ is an eigenstate of $\mathcal{F}_{z}$ with an eigenvalue $m_{F}=-F, \cdots,+F$. Assuming that the local Zeeman energy is minimized for $B_{1}^{\prime}<0, B_{0}>0$, and $g_{F}<0$ we obtain in the $F=1$ case

$$
\boldsymbol{v}_{s}=\frac{\hbar}{m} \frac{1-\cos \vartheta^{\prime}}{r^{\prime} \sin \vartheta^{\prime}} \hat{\boldsymbol{e}}_{\varphi^{\prime}}
$$

where $\left(r^{\prime}, \varphi^{\prime}, \vartheta^{\prime}\right)$ refer to spherical coordinates in the new coordinate system. Similar result has also been obtained in Ref. [19] for the hedgehog texture of Fig. 11 b).

The superfluid velocity $\boldsymbol{v}_{s}$ is equivalent to the vector potential of a magnetic monopole and it has a Dirac string along the negative $z^{\prime}$ axis if $B_{0}(t=0)>0$. For spinor condensates the superfluid flow can be irrotational and it is characterized by its vorticity $\Omega_{s}=\nabla \times \boldsymbol{v}_{s}$ which becomes

$$
\boldsymbol{\Omega}_{s}=\frac{\hbar}{m} \frac{1}{r^{\prime 2}} \hat{e}_{r^{\prime}}
$$

indicating that vorticity corresponding to the imprinted monopole defect is equivalent to the magnetic field of a magnetic monopole, see Fig. 1 In particular, the topology of $\boldsymbol{\Omega}_{s}$ is unaffected by the scaling and translation, and hence $\Omega_{s}$ remains equivalent to the hedgehog texture shown in Fig. 1(b) also in the original coordinate system. The Mermin-Ho relation for general spin- $F[25,26]$ can be computed from Eq. (3) yielding

$$
\boldsymbol{\Omega}_{S}=\frac{m_{F} \hbar}{2 m} \varepsilon_{i j k} \hat{n}_{i} \nabla \hat{n}_{j} \times \nabla \hat{n}_{k} .
$$

In the adiabatic limit spin $\mathcal{S}$ aligns with $\hat{\boldsymbol{n}}$ and the charge $\mathcal{Q}$ in Eq. (2) is thus directly proportional to the flux of $\boldsymbol{\Omega}_{s}$ through a surface $\Sigma$ enclosing the defect. The flux of the monopole is supplied by the Dirac string which is omitted form Eq. (5). In case of $F=1$, the monopole flux is $2 h / m$, that is, two angular momentum quanta, which implies that the vortex filament terminating at the monopole must carry the same amount of vorticity. The situation is thus similar to the Dirac monopole in superfluid ${ }^{3} \mathrm{He}-\mathrm{A}[9,11]$. For a general spin- $F$ BEC the Dirac string carries $2 F$ quanta of angular momentum.

Non-adiabatic effects arising from interactions between atoms, kinetic energy, and the finite timescales in manipulating the external magnetic fields can render the spin to deviate from the direction of the local magnetic field. We take these effects into account by solving the dynamics of the spinor order parameter from the Gross-Pitaevskii mean-field equation [12, 13]

$$
i \hbar \frac{\partial}{\partial t} \boldsymbol{\Psi}=\left[\hat{h}_{0}+c_{0}|\boldsymbol{\Psi}|^{2}+c_{2}\left(\boldsymbol{\Psi}^{\dagger} \mathcal{F} \boldsymbol{\Psi}\right) \cdot \mathcal{F}\right] \boldsymbol{\Psi},
$$

where the interaction strengths $c_{0}$ and $c_{2}$ depend on the scattering lengths in the different channels corresponding to the total hyperfine spin of two scattering particles [13]. The single-particle operator $\hat{h}_{0}$ is given by

$$
\hat{h}_{0}=-\frac{\hbar^{2}}{2 m} \nabla^{2}+V_{\mathrm{opt}}+g_{F} \mu_{B} \boldsymbol{B} \cdot \mathcal{F},
$$

and in the simulation, we employ an external optical potential $V_{\mathrm{opt}}=m r^{2} \omega_{r}^{2} / 2$ and choose the parameters according to ${ }^{87} \mathrm{Rb}$ which implies ferromagnetic interactions.

In the simulation, energy time, and spatial variables are given in the units of $\hbar \omega_{r}, 1 / \omega_{r}$ and $a_{r}=\sqrt{\hbar / m \omega_{r}}$, respectively. For $\omega_{r}=2 \pi \times 250$, the dimensional values of the parameters are given by $B_{1}^{\prime}=-0.05 \mathrm{~T} / \mathrm{m}$ and $B_{0}(t=$ $0)=1 \mu \mathrm{T}$ corresponding to $10^{5}$ atoms. We have also considered a different atom number and antiferromagnetic interactions [33]. The area considered in the simulation is $24 \times 24 \times 27$ in the units of $a_{r}$ and the size of the computational grid varies from $141 \times 141 \times 161$ to $175 \times 175 \times 195$ points. In the simulation, we first calculate the ground state of the system corresponding to the initial values of the magnetic fields using the successive over relaxation (SOR) algorithm, and then propagate the initial state according to the timedependent GP equation (7) using the split operator method 


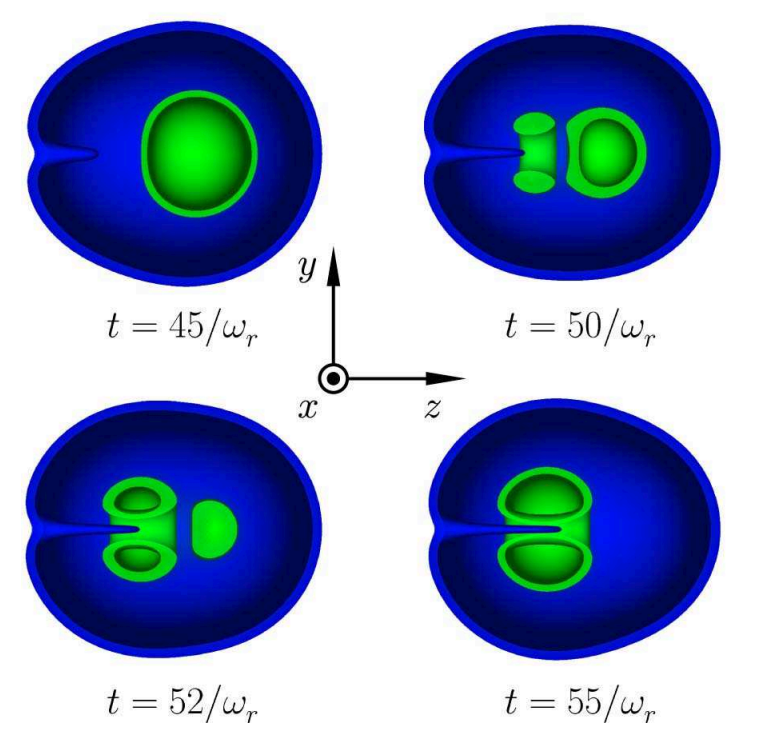

FIG. 2: Particle density at different stages of monopole creation. The monopole is created by ramping the bias field down in a period $t_{0}=$ $50 / \omega_{r}$. Blue (dark) color denotes densities from $\varrho=3.8 \times 10^{-5}$ $N / a_{r}^{3}$ to $\varrho=1.8 \times 10^{-4} N / a_{r}^{3}$ and green (light) color densities from $\varrho=1.4 \times 10^{-3} N / a_{r}^{3}$ to $\varrho=1.5 \times 10^{-3} N / a_{r}^{3}$. The Dirac string is identified as the density depletion that propagates through the condensate.

combined with the Crank-Nicolson method. The time step used in the simulation is $10^{-4} / \omega_{r}$.

Since the Dirac string carries two quanta of angular momentum it is expected to be prone to splitting into two separate strings each carrying one angular momentum quantum. To avoid this scenario, we first imprint the monopole defect with the bias field parallel to $z$ axis. The particle density from this simulation is shown in Fig. 2 The bias field is ramped linearly down to zero in a period $t_{0}=50 / \omega_{r}$ and then equally to negative values. The spin texture $\mathcal{S}$ takes the form described in Fig.11(a) and the corresponding vorticity is shown in Fig. 3 (a). Since the monopole is brought in along the symmetry axis of the system, the Dirac string remains intact. The created monopole appears to be relatively stable: after the bias field was ramped down, the monopole was allowed to evolve in time for a period $5 / \omega_{r}$ in the presence of the two quadrupole fields resulting in slow small-amplitude oscillations along the $z$ axis but it remained otherwise intact. Due to nonadiabatic effects in the creation process, the monopole defect in $\mathcal{S}$ lags slightly behind the zero point of the magnetic field and the Dirac string deviates from a pure $\delta$-function distribution. We have also taken the parameters according to ${ }^{23} \mathrm{Na}$, i.e., corresponding to antiferromagnetic interactions, and obtained essentially the same behavior as in the case of ${ }^{87} \mathrm{Rb}$.

If the rotation symmetry with respect to $z$ axis is explicitly broken by a bias field with $\hat{\boldsymbol{b}} \times \hat{\boldsymbol{z}} \neq 0$, we observe the Dirac string to split into two parts, see Fig 3 (b). The particle density is not depleted all the way along the two strings suggesting that nonadiabatic effects become more pronounced in this (a)

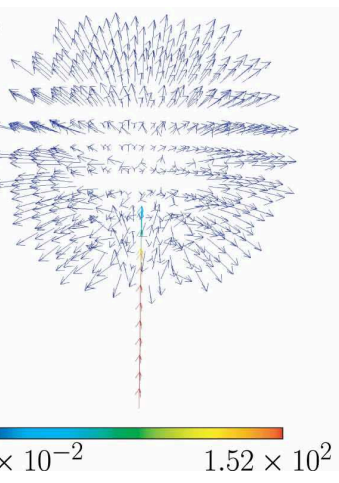

(b)

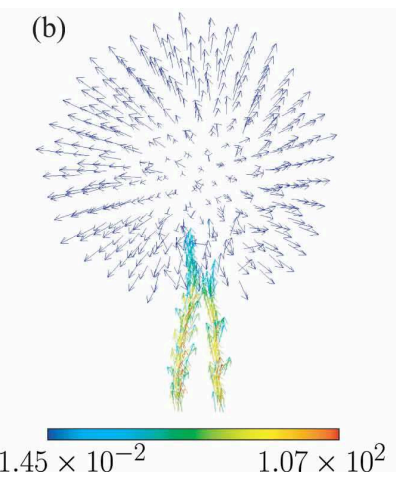

FIG. 3: Vorticity corresponding to the monopole. Vorticity $\boldsymbol{\Omega}_{s}$ is computed numerically after the bias field is ramped from $B_{0}(0)=$ $6.7 \mu \mathrm{T}$ to $B_{0}\left(t_{0}\right)=0$ and it is shown in the units of $h / m a_{r}$. (a) $t_{0}=$ $50 / \omega_{r}$ and the bias field is parallel to $z$ axis. (b) $t_{0}=40 / \omega_{r}$ and the bias field is parallel to the vector $3 / 2 \hat{\boldsymbol{x}}+\hat{\boldsymbol{y}}+14 / 3 \hat{\boldsymbol{z}}$. Magnitude of the vorticity is denoted by color and the map is linear between the minimum and maximum values. For clarity, only the relevant parts of the vector field $\boldsymbol{\Omega}_{s}$ are shown.

case. The spin density $|\mathcal{S}|$, on the contrary, exhibits a clear depletion along the path of the two Dirac strings [32] and together with the state-of-the-art experimental methods [30, 31] it should give an efficient signature of the monopole. In the experiments, a typical imperfection is a slight misalignment and slow drift between the center of the optical trap and the symmetry axis of the magnetic field. We model this by taking $\hat{\boldsymbol{b}} \| \hat{\boldsymbol{z}}$ and adding a small constant term $\tilde{B}_{0} \hat{\boldsymbol{x}}$ to the magnetic field in Eq. (1). This introduces an offset equal to 7\% of the effective radius of the condensate to the path traced by the monopole with respect to $z$ axis. The offset breaks the rotation symmetry of the system but the Dirac string of this off-axis monopole remains undivided (data not shown) suggesting that the instability of the string becomes visible only for fairly large perturbations.

Since the monopole defect in the spin texture is topologically unstable, it can be removed by local surgery. Thus we address to the fate of the monopole after it has been created and all the magnetic fields pinning the monopole are turned off. In the case of superfluid ${ }^{3} \mathrm{He}$, Dirac monopoles are expected to drift to the boundary of the vessel to form a surface defect-boojum [27]. To analyze the decay of the monopole in our case, we have carried out simulations in which the external magnetic fields are switched off immediately or ramped down with constant speed in a period $t_{1}$. For the finite switchoff times, the decay of the monopole initiates already while the external fields are being ramped down. The initial state in the simulation corresponds to a monopole created by ramping the bias field aligned with $z$ axis down in a period $t_{0}=50 / \omega_{r}$.

For ferromagnetic interactions, we have used three different values $t_{1}=0, t_{1}=2.5 / \omega_{r}$ and $t_{1}=5.0 / \omega_{r}$. In all three cases, the qualitative features are the same and they are shown schematically in Fig. 4(see also [32]). For the monopole defect in the spin texture, the monopole unwinds itself along the Dirac string and results in a closed vortex ring, see Fig.4 (a-d). 
In the course of unwinding, a cylindrical domain wall separating the expanding core of the Dirac string from the rest of the texture is formed. Depending on how fast the external fields are turned off, the domain wall is either directly pushed out of the condensate (slow turn-off) or contracted to another vortex ring which eventually drifts out of the condensate (rapid turn-off). In the simulations, the resulting vortex ring in spin texture persists until the end of the simulation which in all three cases spans a period $10 / \omega_{r}$. On the other hand, the unwinding of the monopole in the vorticity $\Omega_{s}$ is depicted in Fig. $4(\mathrm{e}-\mathrm{h})$. During the unwinding of the monopole, vorticity concentrated at the Dirac string diffuses outwards and tends to relax towards more uniformly distributed values.
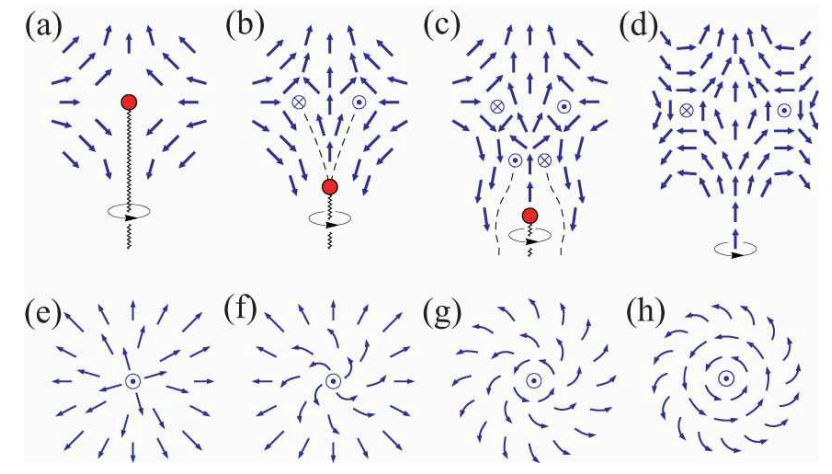

FIG. 4: Unwinding of the monopole defect in the spin texture $\mathcal{S}$ (a)(d) and in the vorticity $\boldsymbol{\Omega}_{S}(\mathrm{e})-(\mathrm{h})$. Arrows pointing upwards (downwards) from the plane are denoted by $\odot(\otimes)$. Along the dashed line in (b) and (c), the spin points either upwards or downwards from the plane. The unwinding in $\boldsymbol{\Omega}_{S}$ is shown in a plane perpendicular to $z$ axis and it is qualitatively independent of the $z$ coordinate. High-resolution figures from the simulation corresponding to these schematic illustrations are available in [32].

For antiferromagnetic interactions we have carried out a simulation with magnetic fields ramped down linearly in a pe$\operatorname{riod} t_{1}=2.5 / \omega_{r}$. The results agree qualitatively with the ferromagnetic case and the end configuration is again a vortex ring in the spin texture. Another vortex ring is generated at the boundary of the condensate and it persists until the end of the simulation. The length of the simulation was $6 / \omega_{r}$ in this case. It should be noted that simulations concerning the unwinding of the monopole span a relatively short period and thus it remains a question for the future research to study if the evolution of the created monopoles differs qualitatively between ferromagnetic and antiferromagnetic interactions. From the global symmetries of the order parameter in the absence of external fields [13, 28], one could expect different behavior by purely topological reasoning [4]. Furthermore, we have not included the possible magnetic dipoledipole interaction which may change the dynamics of the monopole unwinding. In particular, the spin texture considered here resembles the co-called two-z-flare texture that was found to be stabilized by the dipole-dipole interaction [29].

In conclusion, we have introduced and modelled a robust method to create Dirac monopoles in spinor Bose-
Einstein condensates. Our proposal is directly realizable in the present-day experiments and recent developments in the non-destructive imaging of the magnetization of spinor BECs [30, 31] pave way for detecting the exciting dynamics of these monopoles such as the unwinding of the monopole.

Authors acknowledge Jenny and Antti Wihuri Foundation, Emil Aaltonen Foundation, and the Academy of Finland for financial support, and the Center for Scientific Computing Finland (CSC) for computing resources. We thank M. Krusius, G. Volovik, W. Phillips, and P. Cladé for discussions, and J. Huhtamäki for help in numerical calculations.

[1] P. A. M. Dirac, Proc. Roy. Soc. Lond. A 133, 60 (1931).

[2] A. Vilenkin and E. P. S. Shellard, Cosmic Strings and Other Topological Defects (Cambridge University Press, Cambridge, 1994).

[3] A. H. Guth, Phys. Rev. D 23, 347 (1981).

[4] I. Chuang, R. Durrer, N. Turok, and B. Yurke, Science 251, 1336 (1991).

[5] Z. Fang et al., Science 302, 92 (2003).

[6] C. Castelnovo, R. Moessner, and S. L. Sondhi, Nature 451, 42 (2008); L. D. C. Jaubert and P. C. W. Holdsworth, arXiv:0903.1074 (2009).

[7] X.-L. Qi et al. Science 323, 1184 (2009).

[8] S. Blaha, Phys. Rev. Lett. 36, 874 (1976).

[9] G. E. Volovik and V. P. Mineev, JETP. Lett. 23, 593 (1976).

[10] M. M. Salomaa, Nature 326, 367 (1987).

[11] G. E. Volovik, The Universe in a Helium Droplet (Oxford University Press, Oxford, 2003).

[12] T. Ohmi and K. Machida, J. Phys. Soc. Jpn. 67, 1822 (1998).

[13] T.-L. Ho, Phys. Rev. Lett. 81, 742 (1998).

[14] J. Stenger et al., Nature 396, 345 (1998).

[15] J. Ruseckas et al., Phys. Rev. Lett. 95, 010404 (2005).

[16] H. T. C. Stoof, E. Vliegen, and U. Al Khawaja, Phys. Rev. Lett. 87, 120407 (2001); J. P. Martikainen, A. Collin, and K.-A. Suominen, Phys. Rev. Lett. 88, 090404 (2002).

[17] V. Pietilä and M. Möttönen Phys. Rev. Lett. 102, 080403 (2009).

[18] Y. Kawaguchi, M. Nitta, and M. Ueda, Phys. Rev. Lett. 100, 180403 (2008).

[19] C. M. Savage and J. Ruostekoski, Phys. Rev. A 68, 043604 (2003).

[20] J. Ruostekoski and J. R. Anglin, Phys. Rev. Lett. 86, 3934 (2001).

[21] U. Al Khawaja and H. Stoof, Nature 411, 918 (2001).

[22] R. A. Battye, N. R. Cooper, and P. M. Sutcliffe, Phys. Rev. Lett. 88, 080401 (2002); C. M. Savage and J. Ruostekoski, Phys. Rev. Lett. 91, 010403 (2003).

[23] M. Nakahara et al., Physica B 284-288, 17 (2000); T. Isoshima et al., Phys. Rev. A 61, 063610 (2000); S.-I. Ogawa et al., Phys. Rev. A 66, 013617 (2002); M. Möttönen et al., J. Phys.: Condens. Matter 14, 13481 (2002).

[24] A. E. Leanhardt et al., Phys. Rev. Lett. 89, 190403 (2002); A. E. Leanhardt et al., Phys. Rev. Lett. 90, 140403 (2003); Y. Shin et al., Phys. Rev. Lett. 93, 160406 (2004).

[25] T. L. Ho and V. B. Shenoy, Phys. Rev. Lett. 77, 2595 (1996).

[26] A. Lamacraft, Phys. Rev. A 77, 063622 (2008).

[27] N. D. Mermin, Physica B+C 90, 1 (1977).

[28] F. Zhou, Phys. Rev. Lett. 87, 080401 (2001). 
[29] M. Takahashi et al., Phys. Rev. Lett. 98, 260403 (2007).

[30] J. M. Higbie et al., Phys. Rev. Lett. 95, 050401 (2005).

[31] L. E. Sadler et al., Nature 443, 312 (2006).

[32] See the supplementary material for high resolution figures.

[33] For ${ }^{87} \mathrm{Rb}$ we have obtained similar result for $N=5 \times 10^{4}$ atoms. In the case of antiferromagnetic interactions, parameters were taken according to ${ }^{23} \mathrm{Na}$ corresponding to $B_{1}^{\prime}=-0.03$ $\mathrm{T} / \mathrm{m}, B_{0}(t=0)=1 \mu \mathrm{T}$, and $N=4 \times 10^{5}$. 


\title{
Supplementary Figures - Creation of Dirac monopoles in spinor Bose-Einstein condensates
}

\author{
Ville Pietilä $^{1,2}$ and Mikko Möttönen ${ }^{1,2,3}$ \\ ${ }^{1}$ Department of Applied Physics/COMP, Helsinki University of Technology P. O. Box 5100, FI-02015 TKK, Finland \\ ${ }^{2}$ Australian Research Council, Centre of Excellence for Quantum Computer Technology, The University of New South Wales, \\ Sydney 2052, Australia \\ ${ }^{3}$ Low Temperature Laboratory, Helsinki University of Technology, P. O. Box 3500, FI-02015 TKK, Finland
}



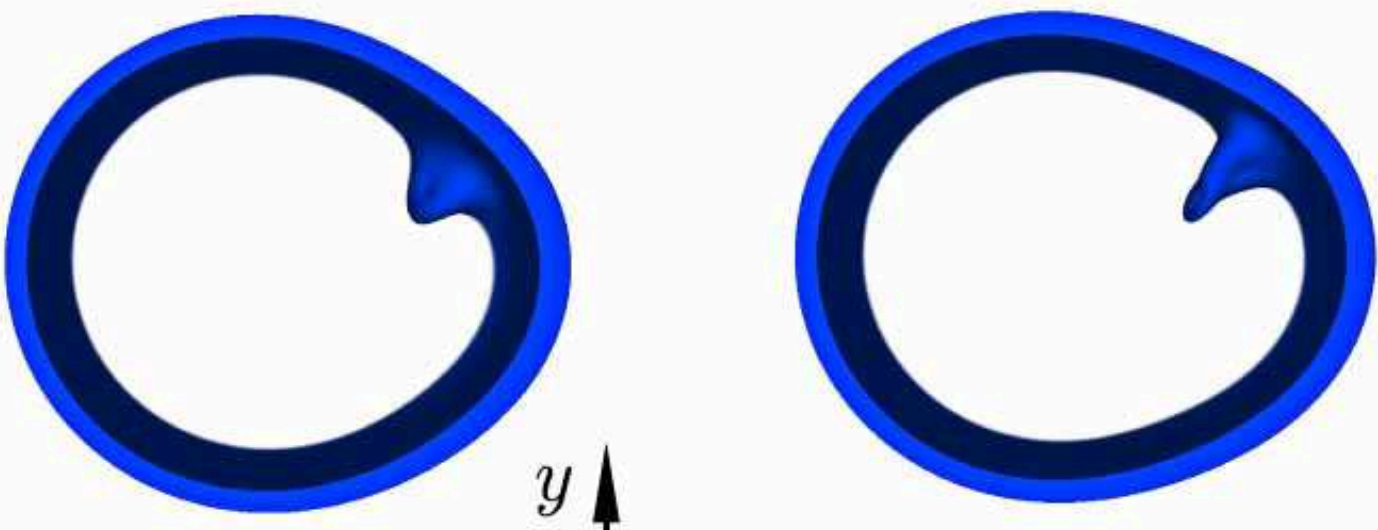

$t=34 / \omega_{r}$

$$
t=36 / \omega_{r}
$$
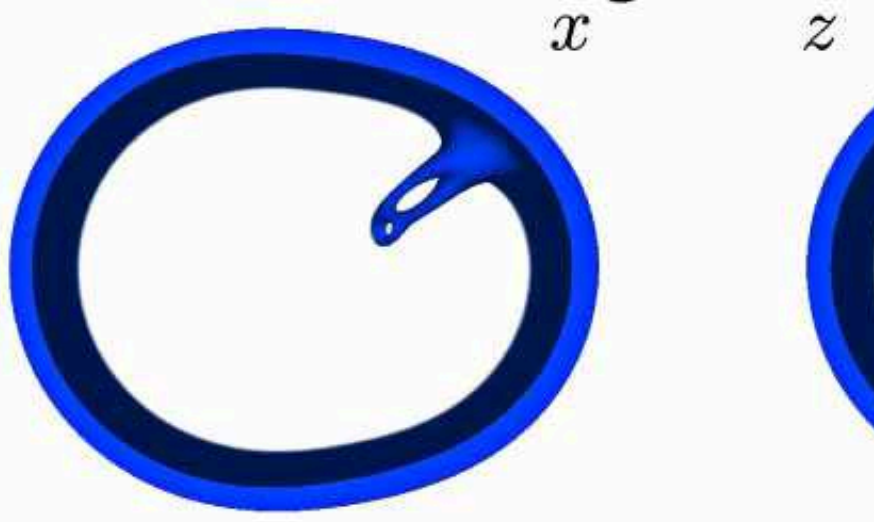

$$
t=38 / \omega_{r}
$$

$$
t=40 / \omega_{r}
$$

FIG. 1: Spin density of the condensate. Splitting of the Dirac string is manifested in the spin density $|\mathcal{S}|$ which is depleted along the two strings. The figure corresponds to spin densities from $|\mathcal{S}|=1.5 \times 10^{-4} \mathrm{~N} / \mathrm{a}_{r}^{3}$ to $|\mathcal{S}|=4.0 \times 10^{-4} \mathrm{~N} / \mathrm{a}_{r}^{3}$. Here $x$ axis points downwards from the plane. 

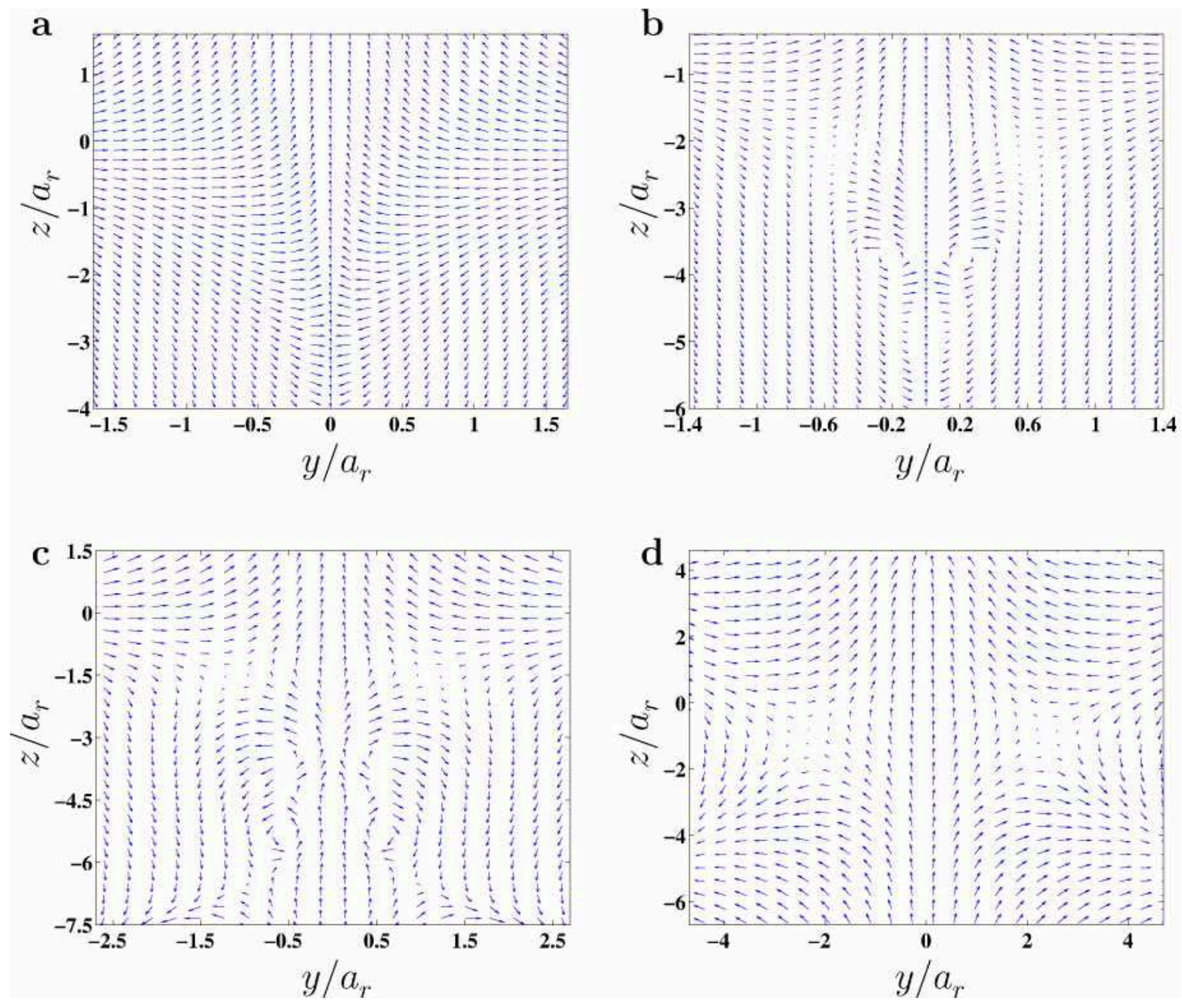

FIG. 2: Unwinding of the monopole defect in the spin texture. The 2D plane corresponds to $x=0$. In the figure, $\hat{s}_{y}$ and $\hat{s}_{z}$ components of the unit spin vector $\hat{s}=\mathcal{S} /|\mathcal{S}|$ are shown. The spatial scale in different panels changes since the radius of the vortex ring changes during its formation. Time corresponding to each panel is $50.5 / \omega_{r}$ (a), $52 / \omega_{r}(b), 53 / \omega_{r}$ (c), and $60 / \omega_{r}$ (d). 
a

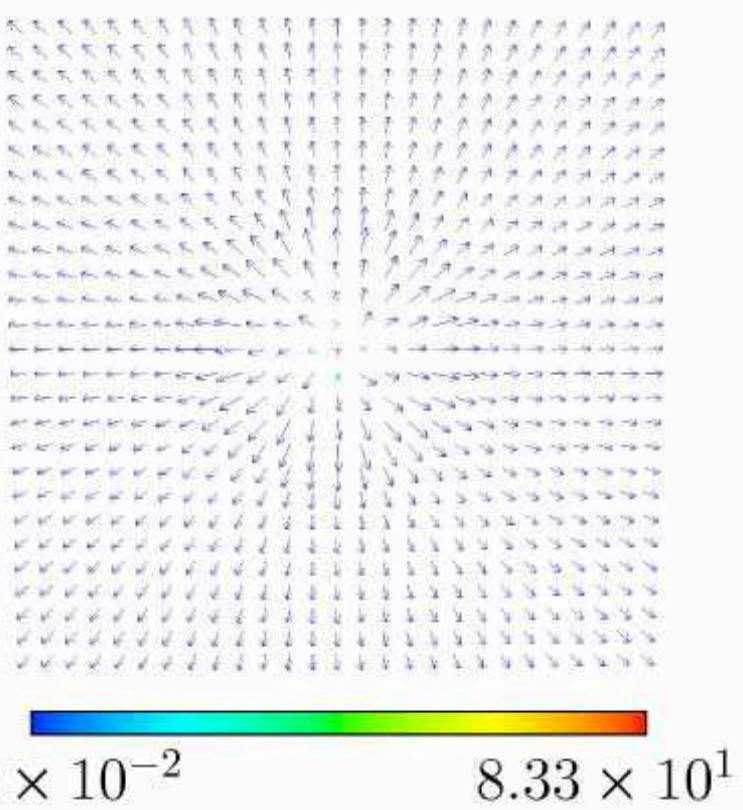

$2.58 \times 10^{-2} \quad 8.33 \times 10^{1}$

C

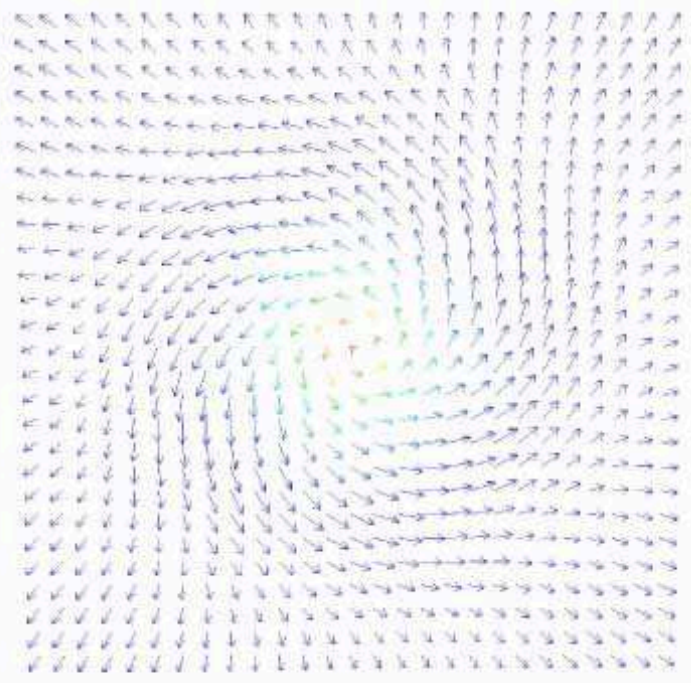

$2.38 \times 10^{-2}$ b

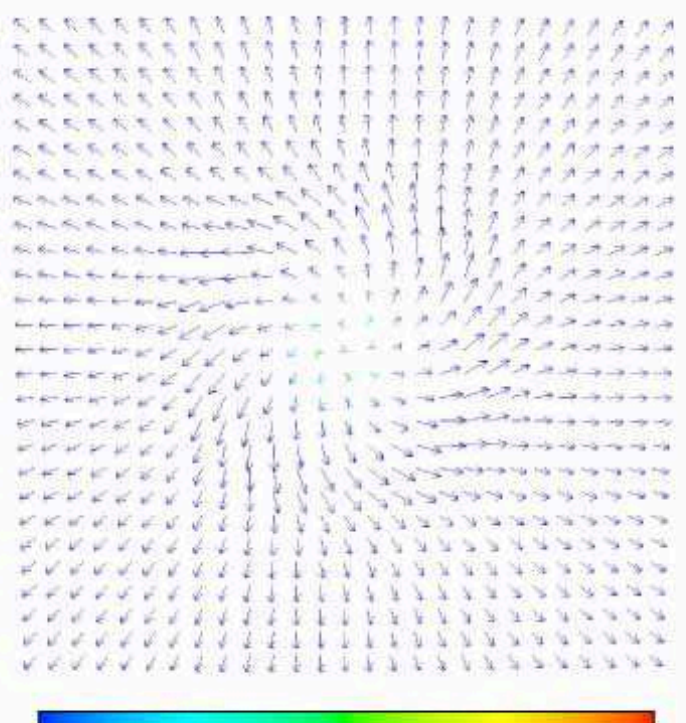

$2.65 \times 10^{-2} \quad 3.00 \times 10^{1}$

d
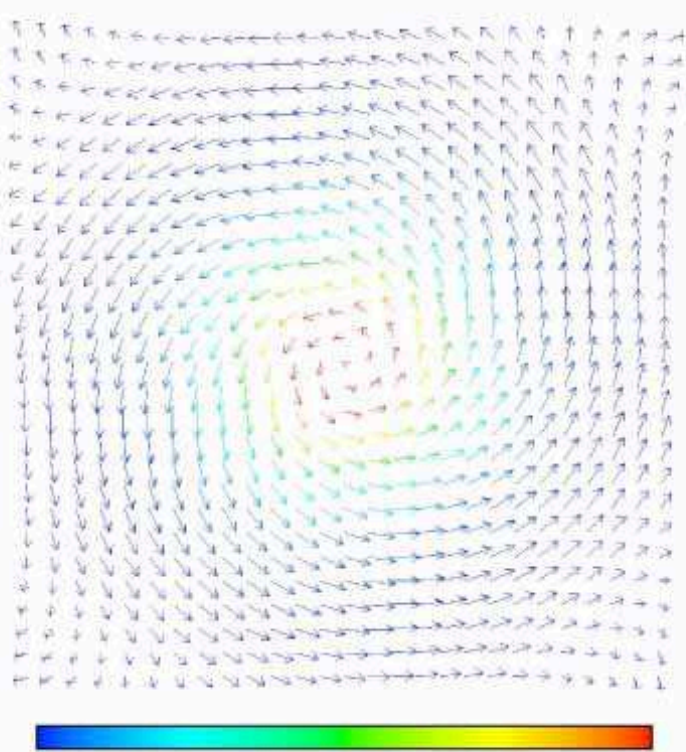

$2.18 \times 10^{-2}$

FIG. 3: Unwinding of the Dirac monopole in vorticity. The 2D plane corresponds to $z=-2.8 a_{r}$. Vorticity is given in the units of $h / m a_{r}$. The time instant corresponding to each panel is $50.5 / \omega_{r}$ (a), $51.5 / \omega_{r}$ (b), $52.5 / \omega_{r}$ (c), and $53.5 / \omega_{r}(\mathrm{~d})$. At the center of the defect, the vector field is always parallel to the $z$ axis pointing upwards from the plane. In the figure $x$ and $y$ axes correspond to horizontal and vertical axes, respectively. The field of view is $15 a_{r} \times 15 a_{r}$. 